\title{
On Erdős-Sós conjecture for trees of large size
}

\author{
Agnieszka Görlich and Andrzej Żak* \\ Faculty of Applied Mathematics \\ AGH University of Science and Technology \\ Kraków, Poland \\ forys@agh.edu.pl, zakandrz@agh.edu.pl
}

Submitted: Jul 13, 2015; Accepted: Mar 3, 2016; Published: Mar 18, 2016

Mathematics Subject Classifications: 05C35

\begin{abstract}
Erdős and Sós conjectured that every graph $G$ of average degree greater than $k-1$ contains every tree of size $k$. Several results based upon the number of vertices in $G$ have been proved including the special cases where $G$ has exactly $k+1$ vertices (Zhou), $k+2$ vertices (Slater, Teo and Yap), $k+3$ vertices (Woźniak) and $k+4$ vertices (Tiner). We further explore this direction. Given an arbitrary integer $c \geqslant 1$, we prove Erdös-Sós conjecture in the case when $G$ has $k+c$ vertices provided that $k \geqslant k_{0}(c)$ (here $k_{0}(c)=c^{12}$ polylog $(c)$ ). We also derive a corollary related to the Tree Packing Conjecture.
\end{abstract}

\section{Introduction}

A set of (simple) graphs $G_{1}, G_{2}, \ldots, G_{q}$ are said to pack into a complete graph $K_{n}$ (in short pack) if $G_{1}, G_{2}, \ldots, G_{q}$ can be found as pairwise edge-disjoint subgraphs in $K_{n}$. Many classical problems in Graph Theory can be stated as packing problems. In particular, $H$ is a subgraph of $G$ if and only if $H$ and the complement of $G$ pack.

Erdős and Sós conjectured that every graph $G$ with average degree greater than $k-1$ contains every tree with $k$ edges. This conjecture has been restated by Woźniak [16] as follows.

Conjecture 1. Suppose that $G$ is a graph with $n$ vertices and $T$ is any tree with $k$ edges. If $|E(G)|<\frac{n(n-k)}{2}$, then $G$ and $T$ pack (into the complete graph $K_{n}$ ).

Ajtai, Komlós, Simonovits and Szemerédi have announced a proof of Conjecture 1 for sufficiently large $\mathrm{k}$. There are many partial results concerning this conjecture. They have been obtained either for some special families of graphs $[2,5,6,15]$ or for some

\footnotetext{
*The authors were partially supported by the Polish Ministry of Science and Higher Education.
} 
special families of trees $[7,11,12]$ or else for certain values of the parameters $k$ and $n$. In particular, the cases where $n$ is equal to $k+1, k+2, k+3$, or $k+4$ were proved by Zhou [17], by Slater, Teo, and Yap [13], by Woźniak [16], and by Tiner [14], respectively. We extend these results to $n=k+c$ for any $c$, provided $k$ is sufficiently large.

Theorem 2. Let $c$ be a positive integer and let $k_{0}(c)=\gamma c^{12} \ln ^{4} c$ where $\gamma$ is some universal sufficiently large constant. Then for every $t=1, \ldots, c$ and for every integer $k \geqslant k_{0}(c)$ the following holds. If $T$ is a tree with $k$ edges and $G$ is a graph on $k+t$ vertices with $|E(G)|<\frac{t(k+t)}{2}$, then $T$ and $G$ pack into $K_{k+t}$.

Another famous tree packing conjecture (TPC) posed by Gyárfás [9] states that any set of $n-1$ trees $T_{n}, T_{n-1}, \ldots, T_{2}$ such that $T_{i}$ has $i$ vertices pack into $K_{n}$. In [8] Bollobás suggested the following weakening of TPC

Conjecture 3. For every $c \geqslant 1$ there is an $n(c)$ such that if $n \geqslant n(c)$, then any set of $c$ trees $T_{1}, T_{2}, \ldots, T_{c}$ such that $T_{i}$ has $n-(i-1)$ vertices pack into $K_{n}$.

Bourgeois, Hobbs and Kasiraj [4] showed that any three trees $T_{n}, T_{n-1}, T_{n-2}$ pack into $K_{n}$. Recently, Balogh and Palmer [3] proved that any set of $t=\frac{1}{10} n^{1 / 4}$ trees $T_{1}, \ldots, T_{t}$ such that no tree is a star and $T_{i}$ has $n-i+1$ vertices pack into $K_{n}$. We obtain the following corollary of Theorem 2 :

Corollary 4. Let $c$ be a positive integer and let $n_{0}(c)=\gamma c^{12} \ln ^{4} c$ where $\gamma$ is some universal sufficiently large constant. If $n \geqslant n_{0}(c)$, then any set of $c$ trees $T_{1}, T_{2}, \ldots, T_{c}$, such that $T_{i}$ has $n-2(i-1)$ vertices pack into $K_{n}$.

Proof. The proof is by induction on $c$. For $c=1$ the statement is obvious. So fix some $c>1$ and assume that the statement is true for $c-1$. Let $T_{1}, T_{2}, \ldots, T_{c}$ be any set of $c$ trees such that $T_{i}$ has $n-2(i-1)$ vertices. By the induction hypothesis $T_{1}, T_{2}, \ldots, T_{c-1}$ pack into $K_{n}$. Let $G$ be a graph with $V(G)=V\left(K_{n}\right)$ and $E(G)=\bigcup_{i=1}^{c-1} E\left(T_{i}\right)$. Clearly,

$$
|E(G)| \leqslant(c-1) n<\frac{(2 c-1) n}{2} .
$$

Furthermore, $T_{c}$ has $n-(2 c-1)$ edges. Thus, by Theorem 2, $G$ and $T_{c}$ pack, which completes the proof of the corollary.

The notation is standard. In particular $|V(G)|$ is called the order of $G$ and $|E(G)|$ is called the size of $G$. Furthermore, $d_{G}(v)$ (abbreviated to $d(v)$ if no confusion arises) denotes the degree of a vertex $v$ in $G, \delta(G)$ and $\Delta(G)$ denote the minimum and the maximum degree of $G$, respectively. $N_{G}(v)$ denotes the set of neighbors of $v$ and, for a subset of vertices $W, N_{G}(W)=\bigcup_{w \in W} N(w) \backslash W$ and $N_{G}[W]=N_{G}(W) \cup W$. 


\section{Preliminaries}

In the proof we refine the approach of Alon and Yuster from [1]. However, we apply it in a slightly different way as we choose random subsets $B_{i}$ (to be defined later) in a denser graph.

We write $\operatorname{Bin}(p, n)$ for the binomial distribution with $n$ trials and success probability $p$. Let $X \in \operatorname{Bin}(n, p)$. We will use the following two versions of the Chernoff bound which follows from formulas (2.5) and (2.6) from [10] by taking $t=2 \mu-n p$ and $t=n p-\mu / 2$, respectively.

If $\mu \geqslant E[X]=n p$ then

$$
\operatorname{Pr}[X \geqslant 2 \mu] \leqslant \exp (-\mu / 3)
$$

On the other hand, if $\mu \leqslant E[X]=n p$ then

$$
\operatorname{Pr}[X \leqslant \mu / 2] \leqslant \exp (-\mu / 8) .
$$

Proposition 5. Let $G$ be a graph with $n$ vertices and at most $m$ edges. Let $V(G)=$ $\left\{v_{1}, \ldots, v_{n}\right\}$ with $d\left(v_{1}\right) \geqslant d\left(v_{2}\right) \geqslant \cdots \geqslant d\left(v_{n}\right)$. Then

$$
d\left(v_{i}\right) \leqslant \frac{2 m}{i} \text {. }
$$

Proof. The proposition is true because

$$
2 m \geqslant \sum_{j=1}^{n} d\left(v_{j}\right) \geqslant \sum_{j=1}^{i} d\left(v_{j}\right) \geqslant i d\left(v_{i}\right) .
$$

The following technical lemma is the main tool in the proof. A version of it appeared in [1].

Lemma 6. Let $G$ be a graph with $n$ vertices and at most $m$ edges. Let $V(G)=\left\{v_{1}, \ldots, v_{n}\right\}$ with $d\left(v_{1}\right) \geqslant d\left(v_{2}\right) \geqslant \cdots \geqslant d\left(v_{n}\right)$. Let $A_{i}, i=1, \ldots, n$, be any subsets of $V(G)$ with the additional requirement that if $u \in A_{i}$ then $d(u)<a$. For $i=1, \ldots, n$ let $B_{i}$ be a random subset of $A_{i}$ where each vertex of $A_{i}$ is independently selected to $B_{i}$ with probability $p<1 / a$. Let

$$
\begin{aligned}
C_{i} & =\left(\bigcup_{j=1}^{i-1} B_{j}\right) \cap N\left(v_{i}\right), \\
D_{i} & =B_{i} \backslash\left(\bigcup_{j=1}^{i-1} N\left[B_{j}\right]\right) .
\end{aligned}
$$

Then

1. $\operatorname{Pr}\left[\left|C_{i}\right| \geqslant 4 m p\right] \leqslant \exp (-2 m p / 3)$ for $i=1, \ldots, n$

2. $\operatorname{Pr}\left[\left|D_{i}\right| \leqslant \frac{p\left|A_{i}\right|}{2 e}\right] \leqslant \exp \left(\frac{-p\left|A_{i}\right|}{8 e}\right)$ for $i=1, \ldots,\lfloor 1 /(a p)\rfloor$. 
Proof. Fix some vertex $v_{i} \in V(G)$.

Consider the first part of the lemma. If $d\left(v_{i}\right) \leqslant 2 m p$ then the probability is zero because $\left|C_{i}\right| \leqslant\left|N\left(v_{i}\right)\right|=d\left(v_{i}\right)$. So we may assume that $d\left(v_{i}\right)>2 m p$. For $u \in N\left(v_{i}\right)$ the probability that $u \in B_{j}$ is at most $p$ (it is either $p$ if $u \in A_{j}$ or 0 if $u \notin A_{j}$.) Thus $\operatorname{Pr}\left[u \in C_{i}\right] \leqslant(i-1) p$. By Proposition $5, i \leqslant 2 m / d\left(v_{i}\right)$. Hence,

$$
\operatorname{Pr}\left[u \in C_{i}\right] \leqslant \frac{2 m p}{d\left(v_{i}\right)}
$$

Observe that $\left|C_{i}\right|$ is a sum of $d\left(v_{i}\right)$ independent indicator random variables each of which has success probability at most $\frac{2 m p}{d\left(v_{i}\right)}$. Thus, the expectation of $\left|C_{i}\right|$ is at most $2 \mathrm{mp}$. Therefore, by (1), the probability of $\left|C_{i}\right|$ being larger than $4 m p$ satisfies

$$
\operatorname{Pr}\left[\left|C_{i}\right| \geqslant 4 m p\right] \leqslant \exp (-2 m p / 3) \text {. }
$$

Consider now the second part of the lemma. Observe that for $u \in A_{i}$, the probability that $u \in B_{i}$ is $p$. On the other hand, for any $j$, the probability that $u \notin N\left[B_{j}\right]$ is at least 1 - ap. Indeed, $u \in N\left[B_{j}\right]$ if and only if $u \in B_{j}$ or one of its neighbors belongs to $B_{j}$. Since $u \in A_{i}$, it has at most $a-1$ neighbors. Hence, the probability that $u \in N\left[B_{j}\right]$ is at most ap. Therefore, as long as $i \leqslant 1 /(a p)$,

$$
\operatorname{Pr}\left[u \in D_{i}\right] \geqslant p(1-a p)^{i-1} \geqslant \frac{p}{e} .
$$

Observe that $\left|D_{i}\right|$ is a sum of $\left|A_{i}\right|$ independent indicator random variables, each having success probability at least $\frac{p}{e}$. Therefore the expectation of $\left|D_{i}\right|$ is at least $\frac{p\left|A_{i}\right|}{e}$. By (2), the probability that $\left|D_{i}\right|$ falls below $\frac{p\left|A_{i}\right|}{2 e}$ satisfies

$$
\operatorname{Pr}\left[\left|D_{i}\right| \leqslant \frac{p\left|A_{i}\right|}{2 e}\right] \leqslant \exp \left(-\frac{p\left|A_{i}\right|}{8 e}\right) .
$$

\section{Proof of Theorem 2}

The proof is by induction on $t$. By Zhou's result the theorem holds for $t=1$. So fix some $t, 2 \leqslant t \leqslant c$, and assume that the statement is true for $t-1$. Let $G^{\prime}$ be a (bipartite) graph that arises from $T$ by adding a set $I^{\prime}$ of $t-1$ isolated vertices. Thus $|V(G)|=\left|V\left(G^{\prime}\right)\right|$. Clearly, $G^{\prime}$ and $G$ pack if and only if $T$ and $G$ pack.

Let $V(G)=\left\{v_{1}, \ldots, v_{n}\right\}$ where $d_{G}\left(v_{i}\right) \geqslant d_{G}\left(v_{i+1}\right)$ and $V\left(G^{\prime}\right)=\left\{v_{1}^{\prime}, \ldots, v_{n}^{\prime}\right\}$ where $d_{G^{\prime}}\left(v_{i}^{\prime}\right) \geqslant d_{G^{\prime}}\left(v_{i+1}^{\prime}\right)$. Since $|E(G)|<t n / 2$, we have

$$
\delta(G) \leqslant t-1
$$

Suppose first that there is a vertex $v \in V(G)$ with $d_{G}(v) \geqslant t+\frac{k-1}{2}$. Clearly,

$$
|E(G-v)|=|E(G)|-d_{G}(v)<\frac{t(k+t)}{2}-t-\frac{k-1}{2}=\frac{(t-1)(k+t-1)}{2} .
$$


Thus, by the induction hypothesis, $G-v$ and $T$ pack. Therefore, $G$ and $T$ pack as well.

Hence, we may assume that

$$
\Delta(G) \leqslant t-1+\frac{k}{2}
$$

Let $S_{i} \subset V(G) \backslash N\left[v_{i}\right]$ with the assumption that if $u \in S_{i}$ then $d_{G}(u)<5 c$.

Claim 7. $\left|S_{i}\right| \geqslant \frac{n}{4}+t$

Proof. By (4) each vertex of $G$ has at least

$$
k+t-1-(t-1+k / 2)=k / 2
$$

non-neighbors. Suppose that $\alpha$ vertices of $G$ have degree greater than or equal to $5 c$. Thus

$$
c n>2|E(G)|=\sum_{i=1}^{n} d\left(v_{i}\right) \geqslant \alpha \cdot 5 c
$$

and so $\alpha \leqslant \frac{n}{5}$. Therefore

$$
\left|S_{i}\right| \geqslant k / 2-\frac{n}{5} \geqslant n / 4+t
$$

Now, we divide the proof into two cases depending whether $\Delta(T)<60 \mathrm{cn}^{3 / 4}$ or $\Delta(T) \geqslant$ $60 \mathrm{cn}^{3 / 4}$.

\subsection{Case $\Delta(T)<60 \mathrm{cn}^{3 / 4}$}

Recall that $S_{i} \subset V(G) \backslash N\left[v_{i}\right]$ with the assumption that if $u \in S_{i}$ then $d_{G}(u)<5 c$.

For $i=1, \ldots, n$ let $B_{i}$ be a random subset of $S_{i}$ where each vertex of $S_{i}$ is independently selected to $B_{i}$ with probability

$$
p=\frac{n^{-3 / 4}}{15 \cdot 10^{2} c^{3}}
$$

Let

$$
\begin{aligned}
C_{i} & =\left(\bigcup_{j=1}^{i-1} B_{j}\right) \cap N\left(v_{i}\right), \\
D_{i} & =B_{i} \backslash\left(\bigcup_{j=1}^{i-1} N\left[B_{j}\right]\right) .
\end{aligned}
$$

Claim 8. The following conditions hold simultaneously with positive probability:

1. $\left|C_{i}\right| \leqslant \frac{n^{1 / 4}}{750 c^{2}}$ for $i=1, \ldots, n$

2. $\left|D_{i}\right| \geqslant 3$ for $i=1, \ldots,\left\lfloor 300 c^{2} n^{3 / 4}\right\rfloor$. 
Proof. Recall that $|E(G)|<\frac{t n}{2} \leqslant \frac{c n}{2}$. Thus, by Lemma 6,

$$
\operatorname{Pr}\left[\left|C_{i}\right| \geqslant \frac{n^{1 / 4}}{750 c^{2}}\right] \leqslant \exp \left(\frac{-n^{1 / 4}}{4500 c^{2}}\right)<\frac{1}{2 n} .
$$

Furthermore, by Claim 7,

$$
3 \leqslant \frac{n^{1 / 4}}{12 \cdot 10^{3} e c^{3}}=\frac{n^{-3 / 4} \cdot(n / 4)}{15 \cdot 10^{2} c^{3} \cdot 2 e}<\frac{p\left|S_{i}\right|}{2 e} .
$$

Hence, by Lemma 6 (with $a=5 c$ and $A_{i}=S_{i}$ ), for each $i \leqslant\lfloor 1 /(a p)\rfloor=\left\lfloor 300 c^{2} n^{3 / 4}\right\rfloor$

$$
\begin{aligned}
\operatorname{Pr}\left[\left|D_{i}\right| \leqslant 3\right] & \leqslant \operatorname{Pr}\left[\left|D_{i}\right| \leqslant \frac{p\left|S_{i}\right|}{2 e}\right] \leqslant \exp \left(-\frac{p\left|S_{i}\right|}{8 e}\right) \\
& \leqslant \exp \left(-\frac{n^{1 / 4}}{48 \cdot 10^{3} e c^{3}}\right)<\frac{1}{600 c^{2} n^{3 / 4}} .
\end{aligned}
$$

Thus, by the union bound, each part of the lemma holds with probability greater than $1 / 2$. Hence both hold with positive probability.

Therefore, we may fix sets $B_{1}, \ldots, B_{n}$ satisfying all the conditions of Claim 8 with respect to the cardinalities of the sets $C_{i}$ and $D_{i}$. We construct a packing $f: V(G) \rightarrow V\left(G^{\prime}\right)$ in three stages. At each point of the construction, some vertices of $V(G)$ are matched to some vertices of $V\left(G^{\prime}\right)$, while the other vertices of $V(G)$ and $V\left(G^{\prime}\right)$ are yet unmatched. Initially, all vertices are unmatched. We always maintain the packing property, that is for any $u, v \in V(G)$ if $u v \in E(G)$ then $f(u) f(v) \notin E\left(G^{\prime}\right)$.

In Stage 1 we match certain number of vertices of $G$ that have the largest degrees. After this stage, by the assumption that $\Delta\left(G^{\prime}\right) \leqslant 60 \mathrm{cn}^{3 / 4}$, both $G$ and $G^{\prime}$ do not have unmatched vertices of high degree (vertices of high degree are the main obstacle in packing). This fact enables us to complete the packing in Stages 2 and 3.

Stage 1 Let $x$ be the largest integer such that $d_{G}\left(v_{x}\right) \geqslant \frac{n^{1 / 4}}{300 c}$. Thus, by Proposition 5 ,

$$
x \leqslant 300 c^{2} n^{3 / 4}
$$

This stage is done repeatedly for $i=1, \ldots, x$ and throughout it we maintain the following two invariants

1. At iteration $i$ we match $v_{i}$ with some vertex $f\left(v_{i}\right)$ of $G^{\prime}$ such that $d_{G^{\prime}}\left(f\left(v_{i}\right)\right) \leqslant 3$.

2. Furthermore, we match all yet unmatched neighbors of $f\left(v_{i}\right)$ to some vertices of $B_{i}$ (this way all neighbors of $f\left(v_{i}\right)$ in $G^{\prime}$ are matched to vertices of $\bigcup_{j=1}^{i} B_{j}$ ).

To see that this is possible, consider the i'th iteration of Stage 1 where $v_{i}$ is some yet unmatched vertex of $G$. Let $Q^{\prime}$ be the set of all yet unmatched vertices of $G^{\prime}$ having degree less than or equal to 3 . Note that, by Proposition 5, the number of vertices of degree less than or equal to 3 in $G^{\prime}$ is at least $n / 2$. Hence,

$$
\left|Q^{\prime}\right| \geqslant n / 2-4(i-1) \geqslant n / 2-4 x \geqslant n / 2-1200 c^{2} n^{3 / 4} \geqslant n / 3 .
$$


Let $X$ be the set of already matched neighbors of $v_{i}$ and let $Y^{\prime}=N_{G^{\prime}}(f(X))$. Thus, the valid choice for $f\left(v_{i}\right)$ would be a vertex of $Q^{\prime} \backslash Y^{\prime}$. To see that such a choice is possible, it is enough to show that $\left|Q^{\prime}\right|>\left|Y^{\prime}\right|$. Let $X=X_{1} \cup X_{2}$ with $X_{1} \subseteq\left\{v_{1}, \ldots, v_{i-1}\right\}$ and $X_{2} \subseteq B_{1} \cup \cdots \cup B_{i-1}$. Hence $X_{1} \leqslant x$ and $\left|X_{2}\right|=\left|C_{i}\right|$. Thus, by the first invariant of Stage 1 , and by (6) and Claim 8

$$
\begin{aligned}
\left|Q^{\prime}\right|-\left|Y^{\prime}\right| & \geqslant n / 3-3\left|X_{1}\right|-\Delta\left(G^{\prime}\right)\left|X_{2}\right| \geqslant n / 3-3 x-60 c n^{3 / 4}\left|C_{i}\right| \\
& \geqslant n / 3-900 c^{2} n^{3 / 4}-60 c n^{3 / 4} \frac{n^{1 / 4}}{750 c^{2}} \\
& =n / 3-6 n /(75 c)-900 c^{2} n^{3 / 4}>n / 4-900 c^{2} n^{3 / 4}>0 .
\end{aligned}
$$

In order to maintain the second invariant it remains to match the yet unmatched neighbors of $f\left(v_{i}\right)$ with vertices from $B_{i}$. Let $R^{\prime}$ be the set of neighbors of $f\left(v_{i}\right)$ in $G^{\prime}$ that are still unmatched. Recall that $\left|R^{\prime}\right| \leqslant 3$. We have to match vertices of $R^{\prime}$ with some vertices of $B_{i}$. Since $D_{i}=B_{i} \backslash\left(\bigcup_{j=1}^{i-1} N\left[B_{j}\right]\right)$, a valid choice of such vertices is by taking an $\left|R^{\prime}\right|$-subset of $D_{i}$. By Claim 8 and by $(6),\left|D_{i}\right| \geqslant 3$ for $i=1, \ldots, x$. Furthermore, since each $v \in D_{i}$ satisfies $d_{G}(v)<5 c \leqslant d_{G}\left(v_{x}\right), D_{i} \cap\left\{v_{1}, \ldots, v_{i-1}\right\}=\emptyset$. Thus, all vertices of $D_{i}$ are still unmatched. Hence, such a choice is possible.

Stage 2 Let $M_{1}$ and $M_{1}^{\prime}$ be the set of matched vertices of $G$ and $G^{\prime}$ after Stage 1, respectively. Clearly $\left|M_{1}\right|=\left|M_{1}^{\prime}\right| \leqslant 4 x<n / 9$. Hence $G^{\prime}-M_{1}^{\prime}$ has an independent set $J^{\prime}$ with $\left|J^{\prime}\right| \geqslant 4 n / 9$.

In Stage 2 we match the vertices from $V\left(G^{\prime}\right) \backslash\left(M_{1}^{\prime} \cup J^{\prime}\right)$, one by one, with some $n-\left|M_{1}\right|-\left|J^{\prime}\right|$ vertices from $V(G) \backslash M_{1}$. Suppose that $v^{\prime} \in V\left(G^{\prime}\right) \backslash\left(M_{1}^{\prime} \cup J^{\prime}\right)$ is still unmatched. Let $Q$ be the set of all yet unmatched vertices of $G$. Clearly, $|Q| \geqslant\left|J^{\prime}\right| \geqslant 4 n / 9$ since the vertices of $J^{\prime}$ remain unmatched in every step of Stage 2. Let $X^{\prime}$ be the set of already matched neighbors of $v^{\prime}$. Let $Y=N_{G}\left(f^{-1}\left(X^{\prime}\right)\right)$. Thus, the valid choice for $f^{-1}\left(v^{\prime}\right)$ would be a vertex of $Q \backslash Y$. To see that such a choice is possible we will prove that $|Q \backslash Y|>0$.

Recall that

$$
\left|X^{\prime}\right| \leqslant 60 \mathrm{cn}^{3 / 4}
$$

What is more, by the second invariant of Stage 1 , the neighbors of each $f\left(v_{i}\right), i=1, \ldots, x$, are already matched. Hence,

$$
X^{\prime} \subset V\left(G^{\prime}\right) \backslash\left\{f\left(v_{1}\right), \ldots, f\left(v_{x}\right)\right\}
$$

and so

$$
f^{-1}\left(X^{\prime}\right) \subset V(G) \backslash\left\{v_{1}, \ldots, v_{x}\right\} .
$$

Thus, by the definition of $x$, for each $u^{\prime} \in X^{\prime}$ we have

$$
\left|N_{G}\left(f^{-1}\left(u^{\prime}\right)\right)\right| \leqslant \frac{n^{1 / 4}}{300 c}
$$


Therefore,

$$
|Q \backslash Y| \geqslant|Q|-\left|X^{\prime}\right| \frac{n^{1 / 4}}{300 c} \geqslant 4 n / 9-60 c n^{3 / 4} \frac{n^{1 / 4}}{300 c}>0 .
$$

Stage 3 Let $M_{2}$ and $M_{2}^{\prime}$ be the sets of matched vertices of $G$ and $G^{\prime}$ after Stage 2 , respectively. In order to complete a packing of $G$ and $G^{\prime}$, it remains to match the vertices of $V(G) \backslash M_{2}$ with the vertices of $J^{\prime}$. Consider a bipartite graph $B$ whose sides are $V(G) \backslash M_{2}$ and $J^{\prime}$. For two vertices $u \in V(G) \backslash M_{2}$ and $v^{\prime} \in J^{\prime}$, we place an edge $u v^{\prime} \in E(B)$ if and only if it is possible to match $u$ with $v^{\prime}$ (by this we mean that mapping $u$ to $v^{\prime}$ will not violate the packing property). Thus $u$ is not allowed to be matched to at most $d_{G}(u) \Delta\left(G^{\prime}\right)$ vertices of $J^{\prime}$. Hence

$$
d_{B}(u) \geqslant\left|J^{\prime}\right|-\frac{n^{1 / 4}}{300 c} 60 c n^{3 / 4}>\left|J^{\prime}\right|-2 n / 9 \geqslant\left|J^{\prime}\right| / 2 .
$$

Now we will evaluate $d_{B}\left(v^{\prime}\right)$. We define $X^{\prime}$ and $Y$ in the same way as in Stage 2. Then (7) holds again. Hence, $v^{\prime}$ is not allowed to be matched to at most $\Delta\left(G^{\prime}\right) \frac{n^{1 / 4}}{300 c}$ vertices of $V(G) \backslash M_{2}$. Thus,

$$
d_{B}\left(v^{\prime}\right) \geqslant\left|J^{\prime}\right|-\frac{n^{1 / 4}}{300 c} 60 c n^{3 / 4} \geqslant\left|J^{\prime}\right| / 2 .
$$

Therefore, by Hall's Theorem there is a perfect matching in $B$, and so a packing of $G$ and $G^{\prime}$.

\subsection{Case $\Delta(T) \geqslant 60 \mathrm{cn} n^{3 / 4}$}

In this case we will follow the ideas from the previous subsection. However, the key difference is that now both $G$ and $G^{\prime}$ may have vertices of high degrees. Because of this obstacle, a packing has two more stages at the beginning. After a preparatory Stage 1, in Stage 2 we match the vertices of $G$ that have high degrees with vertices of $G^{\prime}$ that have small degrees. Then in Stage 3, we match the vertices of $G^{\prime}$ having high degree. This stage is very similar to Stage 1 from the previous subsection, but with the change of the role of $G$ and $G^{\prime}$. Finally, we complete the packing in Stages 4 and 5, which are analogous to Stages 2 and 3 from the previous subsection.

Let

$$
q=\frac{n^{1 / 4}}{59 c}
$$

Let $P^{\prime} \subseteq N_{G^{\prime}}\left(v_{1}^{\prime}\right)$ be the set of neighbors of $v_{1}^{\prime}$ such that each vertex in $P^{\prime}$ has degree at most $q$ in $G^{\prime}$, and every neighbor different from $v_{1}^{\prime}$ of every vertex from $P^{\prime}$ has degree at most $q$ in $G^{\prime}$.

Claim 9. $\left|P^{\prime}\right|>c n^{3 / 4}$. 
Proof. Note that every vertex $v^{\prime} \in N_{G^{\prime}}\left(v_{1}^{\prime}\right) \backslash P^{\prime}$ has the property that $d_{G^{\prime}}\left(v^{\prime}\right)>q$ or $v^{\prime}$ has a neighbor $w^{\prime} \neq v_{1}^{\prime}$ such that $d_{G^{\prime}}\left(w^{\prime}\right)>q$. Therefore,

$$
n=\left|V\left(G^{\prime}\right)\right|>\left(\Delta\left(G^{\prime}\right)-\left|P^{\prime}\right|\right) q \geqslant\left(60 c n^{3 / 4}-\left|P^{\prime}\right|\right) \frac{n^{1 / 4}}{59 c}
$$

and the statement follows.

We construct a packing $f: V(G) \rightarrow V\left(G^{\prime}\right)$ in five stages. At each point of the construction, some vertices of $V(G)$ are matched to some vertices of $V\left(G^{\prime}\right)$, while the other vertices of $V(G)$ and $V\left(G^{\prime}\right)$ are yet unmatched. Initially, all vertices are unmatched.

Stage 1. We first match $v_{n}$ with $v_{1}^{\prime}$, i.e. $f\left(v_{n}\right)=v_{1}^{\prime}$. Next we match the neighbors of $v_{n}$ with $d_{G}\left(v_{n}\right)$ vertices from $I^{\prime}$. This is possible since, by $(3), d_{G}\left(v_{n}\right)=\delta(G) \leqslant t-1=\left|I^{\prime}\right|$. Moreover, since $I^{\prime}$ is a set of isolated vertices, this maping does not violate the packing property.

Stage 2. Let $z$ be the largest integer such that $d_{G}\left(v_{z}\right) \geqslant n^{1 / 4}$. Since $|E(G)|<c n / 2$, by Proposition 5

$$
z \leqslant c n^{3 / 4}
$$

This stage is done repeatedly for $i=1, \ldots, z$ and throughout it we maintain the following invariants:

1. At iteration $i$ we match $v_{i}$ (if it is not matched in Stage 1) with some vertex $f\left(v_{i}\right)$ of $G^{\prime}$ such that $f\left(v_{i}\right) \in P^{\prime} \cup I^{\prime}$.

2. Furthermore, we also make sure that all neighbors of $f\left(v_{i}\right)$ in $G^{\prime}$ are matched to vertices of $S_{i} \cup\left\{v_{n}\right\}$.

Note that because $G^{\prime}$ is acyclic and since there are no edges (in $G$ ) between $v_{i}$ and $S_{i} \cup\left\{v_{n}\right\}$ for those $v_{i}$ that are non-neighbors of $v_{n}$, such a mapping does not violate the packing property.

To see that this mapping is possible, consider the $i$ 'th iteration of Stage 2, where $v_{i}$ is a vertex of $G$ with $d_{G}\left(v_{i}\right) \geqslant n^{1 / 4} \geqslant 5 c$. In particular $v_{i} \notin \bigcup_{j=1}^{i-1} S_{j}$. Thus, if $v_{i}$ is already matched, then it was matched in Stage 1 and so $f\left(v_{i}\right) \in I^{\prime}$. Then, the second invariant of Stage 2 is automatically preserved because $f\left(v_{i}\right)$ is isolated.

Therefore, we may assume that $v_{i}$ is yet unmatched. In this case we may take $f\left(v_{i}\right)$ to be any vertex of $P^{\prime}$. Indeed, note that $\left|P^{\prime}\right| \geqslant z$ and before iteration $i$, the number of already matched vertices of $P^{\prime}$ was at most $i-1$.

Furthermore, observe that since $v_{1}^{\prime}$ is a common neighbor of all $f\left(v_{j}\right), j=1, \ldots, i$, at iteration $i$ the overall number of matched vertices is at most

$$
\delta(G)+1+i q \leqslant t+z q \leqslant t+n / 59 .
$$

Let $R^{\prime}$ be the set of neighbors of $f\left(v_{i}\right)$ in $G^{\prime}$ that are still unmatched. Note that $R^{\prime}$ contains all neighbors of $f\left(v_{i}\right)$ apart from $v_{1}^{\prime}$. Thus, in order to maintain the second invariant, it 
suffices to match vertices of $R^{\prime}$ with some vertices of $S_{i}$. Note that by the choice of $P^{\prime}$ and since $v_{1}^{\prime}$ is already matched, $\left|R^{\prime}\right| \leqslant q-1$. Let $Q$ be the set of yet unmatched vertices of $S_{i}$. By Claim 7 and formula (10),

$$
|Q| \geqslant n / 4+t-(t+n / 59) \geqslant \frac{n^{1 / 4}}{59 c}>q-1 .
$$

Hence, this is possible.

Before we describe Stage 3, we need some preparations. Let $M_{2}$ be the set of all vertices of $G$ that were matched in Stage 1 or 2 . Similarly, let $M_{2}^{\prime}$ be the set of all vertices of $G^{\prime}$ that were matched in Stage 1 or 2. Recall that

$$
\left|M_{2}\right|=\left|M_{2}^{\prime}\right| \leqslant t+z q \leqslant t+n / 59 .
$$

Let $H=G\left[V \backslash M_{2}\right]$ be a subgraph of $G$ induced by yet unmatched vertices. Similarly let $H^{\prime}=G^{\prime}\left[V^{\prime} \backslash M_{2}^{\prime}\right]$. Note that since $G^{\prime}$ is acyclic and by the construction of Stages 1 and 2,

$$
d_{G^{\prime}}\left(v^{\prime}\right) \leqslant d_{H^{\prime}}\left(v^{\prime}\right)+1 \text { for each } v^{\prime} \in V^{\prime} \backslash M_{2}^{\prime} .
$$

Let $V\left(H^{\prime}\right)=\left\{w_{1}^{\prime}, \ldots, w_{r}^{\prime}\right\}$ with $d_{H^{\prime}}\left(w_{1}^{\prime}\right) \geqslant d_{H^{\prime}}\left(w_{2}^{\prime}\right) \geqslant \cdots \geqslant d_{H^{\prime}}\left(w_{r}^{\prime}\right)$. By $(11)$,

$$
r \geqslant n-(t+n / 59)>3 n / 4 \text {. }
$$

Let $y$ be the largest integer such that $d_{H^{\prime}}\left(w_{y}^{\prime}\right) \geqslant 360 \sqrt{n}$. Then, by Proposition 5,

$$
y \leqslant \frac{2 n}{360 \sqrt{n}}=\frac{\sqrt{n}}{180} .
$$

For each $1 \leqslant i \leqslant r$ we define a set $S_{i}^{\prime} \subseteq V\left(H^{\prime}\right) \backslash N_{H^{\prime}}\left[w_{i}^{\prime}\right]$ to be a largest independent set of vertices but with the additional requirement that each $w^{\prime} \in S_{i}^{\prime}$ has $d_{H^{\prime}}\left(w^{\prime}\right)<180$.

Claim 10. $\left|S_{i}^{\prime}\right| \geqslant n / 10$ for $i \geqslant 1$.

Proof. Note that each $w_{i}^{\prime}$ has at least

$$
r-d_{H^{\prime}}\left(w_{i}^{\prime}\right)-1 \geqslant r-d_{G^{\prime}}\left(w_{i}^{\prime}\right)-1 \geqslant r-d_{G^{\prime}}\left(v_{2}^{\prime}\right)-1 \geqslant r-\frac{n}{2}-1 \geqslant \frac{3}{4} n-\frac{n}{2}-1=\frac{n}{4}-1
$$

non-neighbors. Since $H^{\prime}$ is a forest, the subgraph of $H^{\prime}$ induced by all non-neighbors of $w_{i}^{\prime}$ has an independent set of cardinality at least $\frac{n / 4-1}{2}>n / 9$. Let $\alpha$ be the number of vertices of $H^{\prime}$ that have degree greater than or equal to 180 . Thus

$$
2 n>\sum_{j=1}^{r} d_{H^{\prime}}\left(w_{j}^{\prime}\right) \geqslant \alpha \cdot 180,
$$

and so $\alpha \leqslant \frac{n}{90}$. Therefore

$$
\left|S_{i}^{\prime}\right| \geqslant n / 9-\frac{n}{90}=n / 10
$$


For $i=1, \ldots, r$ let $B_{i}^{\prime}$ be a random subset of $S_{i}^{\prime}$ where each vertex of $S_{i}^{\prime}$ is independently selected to $B_{i}^{\prime}$ with probability $1 / \sqrt{n}$. Let

$$
\begin{aligned}
& C_{i}^{\prime}=\left(\bigcup_{j=1}^{i-1} B_{j}^{\prime}\right) \cap N\left(w_{i}^{\prime}\right), \\
& D_{i}^{\prime}=B_{i}^{\prime} \backslash\left(\bigcup_{j=1}^{i-1} N_{H^{\prime}}\left[B_{j}^{\prime}\right]\right) .
\end{aligned}
$$

Claim 11. The following conditions hold simultaneously with positive probability:

1. $\left|C_{i}^{\prime}\right| \leqslant 4 \sqrt{n}$ for $i=1, \ldots, r$

2. $\left|D_{i}^{\prime}\right| \geqslant \frac{\sqrt{n}}{20 e}$ for $i=1, \ldots, y$.

Proof. Clearly, $\left|E\left(H^{\prime}\right)\right|<n$. By Lemma 6 (with $m=n, p=1 / \sqrt{n}$ and $A_{i}=S_{i}^{\prime}$ and $a=180)$,

$$
\operatorname{Pr}\left[\left|C_{i}^{\prime}\right| \geqslant 4 \sqrt{n}\right] \leqslant \exp (-2 \sqrt{n} / 3)<\frac{1}{2 n} \leqslant \frac{1}{2 r} .
$$

Furthermore, by Claim 10,

$$
\frac{\sqrt{n}}{20 e}=\frac{(1 / \sqrt{n})(n / 10)}{2 e} \leqslant \frac{p\left|S_{i}^{\prime}\right|}{2 e} .
$$

Hence, by the second part of Lemma 6 and by (14), for $i \leqslant y \leqslant\lfloor\sqrt{n} / 180\rfloor$ we have

$$
\begin{aligned}
\operatorname{Pr}\left[\left|D_{i}^{\prime}\right| \leqslant \frac{\sqrt{n}}{20 e}\right] & \leqslant \operatorname{Pr}\left[\left|D_{i}^{\prime}\right| \leqslant \frac{p\left|S_{i}^{\prime}\right|}{2 e}\right] \leqslant \exp \left(-\frac{p\left|S_{i}^{\prime}\right|}{8 e}\right) \\
& \leqslant \exp \left(-\frac{(1 / \sqrt{n})(n / 10)}{8 e}\right)=\exp \left(-\frac{\sqrt{n}}{80 e}\right)<\frac{90}{\sqrt{n}} \leqslant \frac{1}{2 y} .
\end{aligned}
$$

Thus, by the union bound, each part of the lemma holds with probability greater than $1 / 2$. Hence both hold with positive probability.

Now we are in the position to describe the next stages of a packing. By Claim 11 we may fix independent sets $B_{1}^{\prime}, \ldots, B_{r}^{\prime}$ satisfying all the conditions of Claim 11 with respect to the cardinalities of the sets $C_{i}^{\prime}$ and $D_{i}^{\prime}$. Let $W=\left\{v_{1}, \ldots, v_{z}\right\}$. Recall that

$$
\Delta(G-W)<n^{1 / 4} \text {. }
$$

Stage 3 This stage is done repeatedly for $i=1, \ldots, y$ and throughout it we maintain the following (similar to those from Stage 2) invariants

1. At iteration $i$ we match $w_{i}^{\prime} \in V\left(H^{\prime}\right)$ with some yet unmatched vertex $u=f^{-1}\left(w_{i}^{\prime}\right)$ of $H$ such that $d_{G}(u) \leqslant 2 c$.

2. Furthermore, we match all yet unmatched neighbors in $H$ of $f^{-1}\left(w_{i}^{\prime}\right)$ to vertices of $B_{i}^{\prime}$ (this way all neighbors of $f^{-1}\left(w_{i}^{\prime}\right)$ in $H$ are matched to vertices of $\bigcup_{j=1}^{i} B_{j}^{\prime}$ ). 
To see that this is possible, consider the i'th iteration of Stage 3 where $w_{i}^{\prime}$ is some yet unmatched vertex of $H^{\prime}$. Let $Q$ be the set of all yet unmatched vertices of $G$ having degree less than or equal to $2 t$. Note that, by Proposition 5 , the number of vertices of degree less than or equal to $2 t$ in $G$ is at least $n / 2$. Hence, by (11) and (14), and since $t \leqslant c$

$$
|Q| \geqslant n / 2-\left|M_{2}\right|-2 t y \geqslant n / 2-t-n / 59-c \sqrt{n} / 90>n / 4
$$

Let $X^{\prime}$ be the set of already matched neighbors (in $G^{\prime}$ ) of $w_{i}^{\prime}$ and let $Y=N_{G}\left(f^{-1}\left(X^{\prime}\right)\right.$ ). Thus, the valid choice for $f^{-1}\left(w_{i}^{\prime}\right)$ would be a vertex of $Q \backslash Y$. Let $X^{\prime}=X_{1}^{\prime} \cup X_{2}^{\prime} \cup X_{3}^{\prime}$ such that $X_{1}^{\prime} \subset M_{2}^{\prime}=V\left(G^{\prime}\right) \backslash V\left(H^{\prime}\right), X_{2}^{\prime} \subset\left\{w_{1}^{\prime}, \ldots, w_{i-1}^{\prime}\right\}$ and $X_{3}^{\prime} \subset \bigcup_{j=1}^{i-1} B_{i}^{\prime}$. By (12), $\left|X_{1}^{\prime}\right| \leqslant 1$. Moreover if $v^{\prime} \in X_{1}^{\prime}$ then, by the second invariant of Stage 2, $v^{\prime} \notin N_{G^{\prime}}\left(v_{1}^{\prime}\right)$. Thus $v^{\prime}=v_{1}^{\prime}$ or $v^{\prime}$ is at distance 2 from $v_{1}^{\prime}$. Hence, either $f^{-1}\left(v^{\prime}\right)=v_{n}$ or $f^{-1}\left(v^{\prime}\right)$ belongs to some set $S_{j}, j \in\{1, \ldots, z\}$. Therefore, $d_{G}\left(f^{-1}\left(v^{\prime}\right)\right) \leqslant 5 c$. Furthermore, $\left|X_{2}^{\prime}\right| \leqslant i-1$ and, by Claim $11,\left|X_{3}^{\prime}\right| \leqslant 4 \sqrt{n}$. Hence, by (15) and by the first invariant of Stage 3 ,

$$
|Y| \leqslant 5 c\left|X_{1}^{\prime}\right|+2 c\left|X_{2}^{\prime}\right|+\left|X_{3}^{\prime}\right| \cdot n^{1 / 4}<n / 4
$$

Therefore, by (16), $|Q \backslash Y|>0$.

In order to maintain the second invariant we have to match yet unmatched neighbors of $f^{-1}\left(w_{i}^{\prime}\right)$ with some vertices of $B_{i}^{\prime}$. Let $R$ be the set of neighbors of $f^{-1}\left(w_{i}^{\prime}\right)$ in $G$ that are still unmatched. Recall that by the first invariant (of Stage 3) $|R| \leqslant 2 c$. Since $D_{i}^{\prime}=$ $B_{i}^{\prime} \backslash\left(\bigcup_{j=1}^{i-1} N\left[B_{j}^{\prime}\right]\right)$, a natural choice of such vertices is taking an $|R|$-subset of $D_{i}^{\prime}$. However, unlike in Stage 1 in the previous subsection, this subset cannot be chosen arbitrarily because of the existence of possible edges between vertices from $P^{\prime \prime}:=N_{G^{\prime}}\left(P^{\prime}\right) \backslash\left\{v_{1}^{\prime}\right\}$ and $D_{i}^{\prime}$. For this reason, we have to match the vertices from $R$ carefully. We match them, one by one, with some vertices from $D_{i}^{\prime}$ in the following way. Suppose that $v \in R$ is yet unmatched. Let $D^{\prime}$ be the set of yet unmatched vertices of $D_{i}^{\prime}$. Since each $w^{\prime} \in D_{i}^{\prime}$ satisfies $d_{H^{\prime}}\left(w^{\prime}\right)<180 \leqslant 360 \sqrt{n}, D_{i}^{\prime} \cap\left\{w_{1}^{\prime}, \ldots, w_{i-1}^{\prime}\right\}=\emptyset$. Hence,

$$
\left|D^{\prime}\right| \geqslant\left|D_{i}^{\prime}\right|-|R| \geqslant \sqrt{n} /(20 e)-2 c .
$$

Let $X_{2}$ be the set of those already matched neighbors $u$ of $v \in R$ which satisfy $f(u) \in P^{\prime \prime}$. Let $Y_{2}^{\prime}=N_{G^{\prime}}\left(f\left(X_{2}\right)\right)$. Thus, the valid choice for $f(v)$ would be a vertex from $D^{\prime} \backslash Y_{2}^{\prime}$. Recall, that by the definition of $z,\left|X_{2}\right| \leqslant d_{G}(v) \leqslant n^{1 / 4}$. Furthermore, by the definition of $P^{\prime}, N_{G^{\prime}}(f(u)) \leqslant q$. Thus, by (8) and (18),

$$
\left|D^{\prime} \backslash Y_{2}^{\prime}\right|>\sqrt{n} /(20 e)-2 c-\sqrt{n} / 59>0 .
$$

Thus, an appropriate choice for $f(v)$ is possible.

Stage 4 Let $M_{3}$ be the set of matched vertices of $G$ after Stage 3. Similarly, let $M_{3}^{\prime}$ be the set of matched vertices of $G^{\prime}$ after Stage 3. Note that, by (14) and (11),

$$
\left|M_{3}\right|=\left|M_{3}^{\prime}\right| \leqslant\left|M_{2}\right|+(2 c+1) y \leqslant t+n / 59+(2 c+1) \sqrt{n} / 180<n / 4
$$


Let $W^{\prime}=\left\{w_{1}^{\prime}, \ldots, w_{y}^{\prime}\right\} \cup\left\{v_{1}^{\prime}\right\}$. By $(12)$,

$$
\Delta\left(G^{\prime}-W^{\prime}\right) \leqslant \Delta\left(H^{\prime}-W^{\prime}\right)+1 \leqslant 360 \sqrt{n}+1 .
$$

Furthermore, $\left|V\left(G^{\prime}\right) \backslash M_{3}^{\prime}\right| \geqslant n-n / 4=3 n / 4$. Thus $G^{\prime}-M_{3}^{\prime}$ has an independent set $J^{\prime}$ with $\left|J^{\prime}\right| \geqslant 3 n / 8$. Let $K^{\prime}=V\left(G^{\prime}\right) \backslash\left(J^{\prime} \cup M_{3}^{\prime}\right)$.

In Stage 4 we match vertices from $K^{\prime}$ one by one, with arbitrary yet unmatched vertices of $G$. Suppose that $v^{\prime} \in K^{\prime}$ is still unmatched. Let $Q$ be the set of all yet unmatched vertices of $G$. Clearly, $|Q| \geqslant\left|J^{\prime}\right| \geqslant 3 n / 8$ since the vertices of $J^{\prime}$ remain unmatched in every step of Stage 4. Let $X^{\prime}$ be the set of already matched neighbors of $v^{\prime}$. Let $Y=N_{G}\left(f^{-1}\left(X^{\prime}\right)\right)$. Thus, the valid choice for $f^{-1}\left(v^{\prime}\right)$ would be a vertex of $Q \backslash Y$. By (20), $\left|X^{\prime}\right| \leqslant 360 \sqrt{n}+1$. Furthermore, by the second invariant of Stage 2,

$$
X^{\prime} \subseteq V\left(G^{\prime}\right) \backslash\left\{f\left(v_{1}\right), \ldots, f\left(v_{z}\right)\right\}
$$

and so

$$
f^{-1}\left(X^{\prime}\right) \subseteq V(G) \backslash W
$$

Hence, by (15),

$$
|Y| \leqslant\left|X^{\prime}\right| \cdot n^{1 / 4}<3 n / 8
$$

Hence

$$
|Q \backslash Y|>0,
$$

and so the choice for $f^{-1}\left(v^{\prime}\right)$ is possible.

Stage 5 Let $M_{4}$ and $M_{4}^{\prime}$ be the sets of matched vertices of $G$ and $G^{\prime}$, respectively, after Stage 4. In order to complete a packing of $G$ and $G^{\prime}$ it remains to match the vertices of $J^{\prime}$ with the yet unmatched vertices of $G$. Consider a bipartite graph $B$ whose sides are $V(G) \backslash M_{4}$ and $J^{\prime}$. For two vertices $u \in V(G) \backslash M_{4}$ and $v^{\prime} \in J^{\prime}$, we place an edge $u v^{\prime} \in E(B)$ if and only if it is possible to match $u$ with $v^{\prime}$ (by this we mean that mapping $u$ to $v^{\prime}$ will not violate the packing property). Recall that, by $(15), d_{G}(u) \leqslant n^{1 / 4}$. Moreover, by the construction of Stage 1 and by the second invariant of Stage 3, $f\left(N_{G}(u)\right) \subset V\left(G^{\prime}\right) \backslash W^{\prime}$. Thus, by (20), $u$ is not allowed to be matched to at most $n^{1 / 4}(360 \sqrt{n}+1)$ vertices of $J^{\prime}$. Therefore,

$$
d_{B}(u) \geqslant\left|J^{\prime}\right|-n^{1 / 4}(360 \sqrt{n}+1)>\left|J^{\prime}\right|-3 n / 16 \geqslant\left|J^{\prime}\right| / 2
$$

Similarly, by $(20), d_{G^{\prime}}\left(v^{\prime}\right) \leqslant 360 \sqrt{n}+1$. Moreover, by the second invariant of Stage 2 , $f^{-1}\left(N_{G^{\prime}}\left[v^{\prime}\right]\right) \subset V(G) \backslash W$. Therefore, by (15),

$$
d_{B}\left(v^{\prime}\right) \geqslant\left|J^{\prime}\right|-n^{1 / 4}(360 \sqrt{n}+1)>\left|J^{\prime}\right| / 2 .
$$

Therefore, by Hall's Theorem there is a perfect matching in $B$, and so a packing of $G$ and $G^{\prime}$. This completes the inductive step, and so the theorem is proved.

\section{Acknowledgements}

We thank the reviewers for carefully reading our manuscript and for giving detailed comments and suggestions that have been helpful to improve the manuscript. 


\section{References}

[1] N. Alon and R. Yuster. The Turán number of sparse spanning graphs. J. Comb. Theory Ser. B, 103(3):337-343, 2013.

[2] S. Balasubramanian and E. Dobson. Constructing trees in graphs with no $K_{2, s}$. J. Graph Theory, 56:301-310, 2007.

[3] J. Balogh and C. Palmer. On the Tree Packing Conjecture. SIAM J. Discrete Math., 27(4):1995-2006, 2013.

[4] B.A. Bourgeois, A.M. Hobbs, and J. Kasiraj. Packing trees in complete graphs. Discrete Math., 67:27-42, 1987.

[5] E. Dobson. Constructing trees in graphs whose complement has no $K_{2, s}$. Combin. Probab. Comput., 11:343-347, 2002.

[6] N. Eaton and G. Tiner. On the Erdős-Sós conjecture for graphs having no path with $k+4$ vertices. Discrete Math., 313:1621-1629, 2013.

[7] G. Fan and L. Sun. The Erdős-Sós conjecture for spiders. Discrete Math., 307:30553062, 2007.

[8] R.L. Graham, M. Grötschel, and L. Lovász, Eds. Handbook of combinatorics. Vol. 1,2. Elsevier Science B.V., Amsterdam, 1995.

[9] A. Gyárfás and J. Lehel. Packing trees of different order into $K_{n}$. In Combinatorics (Proc. Fifth Hungarian Colloq., Keszthely, 1976), Colloq. Math. Soc. János Bolyai, volume 18 pages 463-469, North-Holland, Amsterdam, 1978.

[10] S. Janson, T. Łuczak, and A. Ruciński. Random graphs. Wiley-Interscience Series in Discrete Mathematics and Optimization, Wiley-Interscience, New York, 2000.

[11] A. McLennan. The Erdős-Sós conjecture for trees of diameter four. J.Graph Theory, 49(4):291-301, 2005.

[12] A.F. Sidorenko. Asymptotic solution for a new class of forbidden graphs. Combinatorica, 9(2):207-215, 1989.

[13] P. Slater, S. Teo, and H. Yap. Packing a Tree with a Graph of the Same Size. J. Graph Theory, 9:213-216, 1985.

[14] G. Tiner. On the Erdős-Sós Conjecture for Graphs on $n=k+3$ Vertices. Ars Combin., 95:143-150, 2010.

[15] J.F. Saclé, M. Woźniak. The Erdős-Sós Conjecture for graphs without $C_{4}$. J. Combin. Theory Ser. B, 50:367-372, 1997.

[16] M. Woźniak. On the Erdős-Sós Conjecture. J. Graph Theory, 21:229-234, 1996.

[17] B. Zhou. A note on the Erdős-Sós Conjecture. Acta Math. Sci., 4:287-289, 1984. 